\title{
Preclinical assessment of early tumor response after irradiation by positron emission tomography with 2 -amino-[3- $\left.{ }^{11} \mathrm{C}\right]$ isobutyric acid
}

\author{
ATSUSHI B. TSUII ${ }^{1}$, AYA SUGYO ${ }^{1}$, HITOMI SUDO ${ }^{1}$, CHIE SUZUKI $^{1}$, HIDEKATSU WAKIZAKA $^{2}$, \\ MING-RONG ZHANG ${ }^{3}$, KOICHI KATO ${ }^{3,4}$ and TSUNEO SAGA ${ }^{1}$ \\ ${ }^{1}$ Diagnostic Imaging Program, ${ }^{2}$ Biophysics Program and ${ }^{3}$ Molecular Probe Program, Molecular Imaging Center, \\ National Institute of Radiological Sciences, Inage-ku, Chiba 263-8555; ${ }^{4}$ Department of Integrative Brain Imaging, \\ National Center of Neurology and Psychiatry, Kodaira, Tokyo 187-5551, Japan
}

Received December 2, 2014; Accepted January 9, 2015

DOI: $10.3892 /$ or.2015.3868

\begin{abstract}
The positron emission tomography (PET) probe, 2-amino-[3- $\left.{ }^{11} \mathrm{C}\right]$ isobutyric acid $\left(\left[3-{ }^{11} \mathrm{C}\right] \mathrm{AIB}\right)$, is reported to accumulate less in inflammatory lesions than 2-deoxy-2- $\left[{ }^{18} \mathrm{~F}\right]$ fluoro-D-glucose $\left(\left[{ }^{18} \mathrm{~F}\right] \mathrm{FDG}\right)$ and has the potential for evaluation of the efficacy of radiotherapy. To determine whether $\left[3-{ }^{11} \mathrm{C}\right] \mathrm{AIB}$ is useful to monitor early metabolic change in tumors after radiotherapy, we evaluated the temporal change in [3- $\left.{ }^{11} \mathrm{C}\right] \mathrm{AIB}$ tumor uptake, tumor volume, histological features and expression of amino acid transporters early after radiotherapy in a mouse tumor model. PET with $\left[3-{ }^{11} \mathrm{C}\right] \mathrm{AIB}$ was conducted in mice bearing a subcutaneous tumor (SY, derived from small cell lung cancer) in two schedules: schedule 1, before (day -1) and after (days 1 and 3) 15 Gy of radiation and schedule 2, days $-1,1$ and 5 . $\left[3-{ }^{11}\right.$ C]AIB tumor uptake tended to increase on day 1 after irradiation and decreased thereafter. Tumor uptake was not correlated with tumor volume in schedule 1. Although tumor uptake was correlated with tumor volume in schedule 2 , this correlation was lost when the day 5 data of greatly reduced tumor volumes were excluded. In a separate group of tumor-bearing mice, excised tumor sections were stained with terminal deoxynucleotidyl transferase-mediated deoxyuridine triphosphate nick-end labeling (TUNEL) or anti-Ki-67 antibody. There was no correlation between tumor uptake and percentages of TUNEL- or Ki-67-positive cells. Expression of amino acid transporters, SLC38A1, SLC38A2 and SLC38A4, was determined by real-time RT-PCR. SLC38A1 and SLC38A2 were expressed in SY tumors, and a significant correlation was observed between $\left[3-{ }^{11} \mathrm{C}\right] \mathrm{AIB}$ tumor uptake and SLC38A1 expression. In conclusion, early
\end{abstract}

Correspondence to: Dr Atsushi B. Tsuji, Diagnostic Imaging Program, Molecular Imaging Center, National Institute of Radiological Sciences, 4-9-1 Anagawa, Inage-ku, Chiba 263-8555, Japan E-mail: a_tsuji@nirs.go.jp

Key words: aminoisobutyric acid, imaging, X-rays, radiotherapy, metabolic response, tumor mouse model, amino acid transporter change in $\left[3-{ }^{11} \mathrm{C}\right] \mathrm{AIB}$ tumor uptake after irradiation reflected the temporal change in amino acid transporter expression, while it was independent of change in tumor volume, apoptosis and cell proliferation. PET with $\left[3-{ }^{11} \mathrm{C}\right] \mathrm{AIB}$ has the potential for use in non-invasive evaluation of early metabolic change after irradiation before morphological change of tumors.

\section{Introduction}

In addition to surgery and chemotherapy, radiotherapy is commonly used to treat a wide variety of cancers, although the therapeutic response is known to be varied in each cancer $(1,2)$. Precise evaluation of the efficacy of therapy including radiotherapy requires non-invasive diagnostic methods to evaluate whole tumor regions (2). Positron emission tomography (PET) can non-invasively provide functional information of interest, including data on proliferation and metabolism of tumors, thereby providing information on temporal pathophysiological changes in tumors after therapy (2). PET with 2-deoxy-2- $\left[{ }^{18} \mathrm{~F}\right]$ fluoro-D-glucose $\left(\left[{ }^{18} \mathrm{~F}\right] \mathrm{FDG}\right)$ can evaluate therapeutic efficacy before morphologic change as determined by computed tomography (CT) and magnetic resonance imaging (MRI) and it has been increasingly recognized to be useful for therapeutic response evaluation $(3,4)$. Since increased glucose metabolism is not specific to cancer cells, $\left[{ }^{18} \mathrm{~F}\right] \mathrm{FDG}$ highly accumulates in inflammatory and granulomatous lesions $(5,6)$. When patients have accompanying unexpected/subclinical inflammation, $\left[{ }^{18} \mathrm{~F}\right]$ FDG PET sometimes gives false-positive results. Since radiotherapy often causes inflammatory reactions in irradiated areas $(1,7)$, high $\left[{ }^{18} \mathrm{~F}\right] \mathrm{FDG}$ uptake has been reported to be observed in irradiated areas $(2,7)$. It could be difficult to distinguish inflammation from a metabolically active residual tumor, and it is generally necessary to wait several months after the end of radiotherapy to minimize the influence of radiation-induced inflammation before examination of radiotherapy efficacy. Therefore, the introduction of tracers that are less affected by inflammation than $\left[{ }^{18} \mathrm{~F}\right] \mathrm{FDG}$ is required to complement the limitation of $\left[{ }^{18} \mathrm{~F}\right] \mathrm{FDG}$ PET.

Amino acids are generally required nutrients for proliferating tumor cells and their pooling increases by upregulation of their transporters in many tumor types, while inflammatory cells have a low protein metabolism compared with glucose 
metabolism $(2,8)$. Amino acid PET tracers are known to accumulate less in inflammatory lesions than $\left[{ }^{18} \mathrm{~F}\right] \mathrm{FDG}(8)$. However, one of the most widely used amino acid PET tracers, [methyl- ${ }^{11} \mathrm{C}$ ]methionine, is metabolized in cells, resulting in numerous radiolabeled metabolites, which could decrease tumor specificity and complicate the interpretation of PET data (8). Therefore, several radiolabeled non-natural amino acids that are resistant to in vivo metabolism are expected to be more useful for the above-mentioned purpose (8). We recently demonstrated that 2-amino-[3- $\left.{ }^{11} \mathrm{C}\right]$ isobutyric acid $\left(\left[3-{ }^{11} \mathrm{C}\right]\right.$ AIB), a PET tracer based on a non-natural amino acid, is highly accumulated in tumors, but less in inflammatory lesions compared with $\left[{ }^{18} \mathrm{~F}\right] \mathrm{FDG}$ in a mouse model $(9) .\left[3{ }^{-11} \mathrm{C}\right] \mathrm{AIB}$ has the potential to complement the limitation of $\left[{ }^{18} \mathrm{~F}\right] \mathrm{FDG}$ and more precisely evaluate the efficacy of radiotherapy in patients with unexpected/subclinical inflammation. To date, there is no study evaluating the tumor uptake change of $\left[3-{ }^{11} \mathrm{C}\right] \mathrm{AIB}$ after irradiation in either patients or animal models. In the present study, the early change of $\left[3{ }^{-11} \mathrm{C}\right] \mathrm{AIB}$ uptake in tumors by effective radiotherapy was evaluated. To clarify the contributing factor in the change in $\left[3-{ }^{11} \mathrm{C}\right] \mathrm{AIB}$ uptake, serial quantitative PET with $\left[3{ }^{-11} \mathrm{C}\right] \mathrm{AIB}$ was conducted to quantify tumor uptake; then tumor uptake was compared with the changes in tumor volume, histological features and the expression of amino acid transporters in a mouse model bearing a subcutaneous tumor before and early after X-ray irradiation.

\section{Materials and methods}

Tumor model, X-ray irradiation and PET. A human small cell lung cancer line SY (Immuno-Biological Laboratories, Takasaki, Japan) was maintained in RPMI-1640 (Sigma, St. Louis, MO, USA) containing 5\% fetal bovine serum (Sigma) in a humidified incubator maintained at $37^{\circ} \mathrm{C}$ with $5 \% \mathrm{CO}_{2}$. The animal experimental protocol was approved by the Animal Care and Use Committee of the National Institute of Radiological Sciences, and all the animal experiments were conducted in accordance with the institutional guidelines regarding animal care and handling. Male nude mice (BALB/c-nu/nu, 6-weeks old; Clea Japan, Tokyo, Japan) were maintained under specific pathogen-free conditions. Two million SY cells were subcutaneously injected into a hindlimb under isoflurane anesthesia. When subcutaneous tumors reached a diameter of $\sim 10 \mathrm{~mm}$, the tumors were irradiated with a single dose $15 \mathrm{~Gy}$ of $200 \mathrm{kVp} \mathrm{X}$-rays at a rate of $0.98 \mathrm{~Gy} / \mathrm{min}$ using a Pantak HF-320 X-ray generator (Shimadzu, Kyoto, Japan). Other parts of the mouse body were protected by a brass shield to limit unnecessary radiation exposure. $\left[3-{ }^{11} \mathrm{C}\right] \mathrm{AIB}$ (radiochemical purity $>99 \%$ ) was synthesized from iodo $\left[{ }^{11} \mathrm{C}\right]$ methane and methyl $\mathrm{N}$-(diphenylmethylen)-D,L-alaniate using tetrabutylammonium fluoride-promoted $\alpha$-[11C]methylation (10). PET studies were conducted in two schedules, schedule $1(\mathrm{n}=5)$ and schedule $2(\mathrm{n}=6)$. The time-points of PET scan of schedule 1 were one day before irradiation (day -1) and one and three days after irradiation (days 1 and 3), and those of schedule 2 were days -1, 1 and 5 (Fig.1). The 10-min PET scans were conducted at $30 \mathrm{~min}$ after intravenous injection of $\sim 11.1 \mathrm{MBq}$ of $\left[3-{ }^{11} \mathrm{C}\right] \mathrm{AIB}$ using the small-animal PET system (Inveon; Siemens Medical Solutions, Malvern, PA, USA) under isoflurane anesthesia. Body temperature was maintained
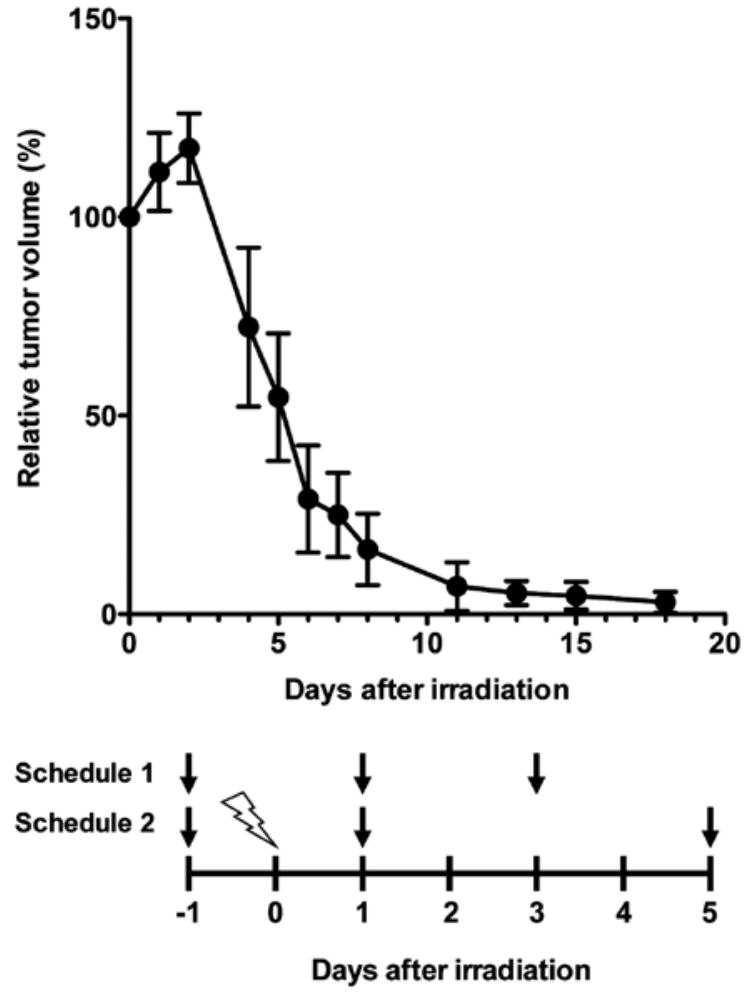

Figure 1. Growth curve of tumors irradiated with 15 Gy X-rays (upper panel). Data are expressed as the mean \pm SD $(n=3)$. The schedules of serial PET with $\left[3-{ }^{11} \mathrm{C}\right] \mathrm{AIB}$ in tumor-bearing mice treated with $15 \mathrm{~Gy} \mathrm{X}$-rays (lower panel). Arrows indicate PET experiments. PET, positron emission tomography.

around $37^{\circ} \mathrm{C}$ by a heating lamp and warm water during scans. Images were reconstructed using a 3D maximum a posteriori (18 iterations with 16 subsets, $\beta=0.2$ ) without attenuation correction. The region of interest (ROI) was manually drawn over tumors and tracer uptake was quantified as the standardized uptake value (SUV)max.

Histological analysis. As a separate experiment, tumors $(\mathrm{n}=5$ for each time-point) were fixed in $10 \%(\mathrm{v} / \mathrm{v})$ neutral buffered formalin and embedded in paraffin for sectioning. Sections $(0.1 \mu \mathrm{m}$ thickness) were stained with hematoxylin and eosin (H\&E). Apoptotic cells were detected by terminal deoxynucleotidyl transferase-mediated deoxyuridine triphosphate nick-end labeling (TUNEL) staining using an (Merck Millipore, Billerica, MA, USA). Ki-67 staining was conducted using an anti-Ki-67 antibody (MIB-1; Dako, Glostrup, Denmark) as described previously (11). TUNEL- and Ki-67positive cells were quantified in at least five randomly selected fields of each section at $\mathrm{x} 400$ magnification.

Real-time quantitative RT-PCR. As a separate experiment, first-strand cDNAs from three tumors were synthesized using a FastLane Cell cDNA kit (Qiagen, Hilden, Germany). Real-time RT-PCR was conducted in triplicate with predesigned and preoptimized TaqMan probes to detect three system A transporters (SLC38A1, SLC38A2, and SLC38A4) and 18S rRNA (Applied Biosystems, Foster City, CA, USA) using Mx3000P qPCR systems (Agilent Technologies, Santa Clara, CA, USA). Gene expression levels were normalized to $18 \mathrm{~S}$ rRNA expression in each sample. 


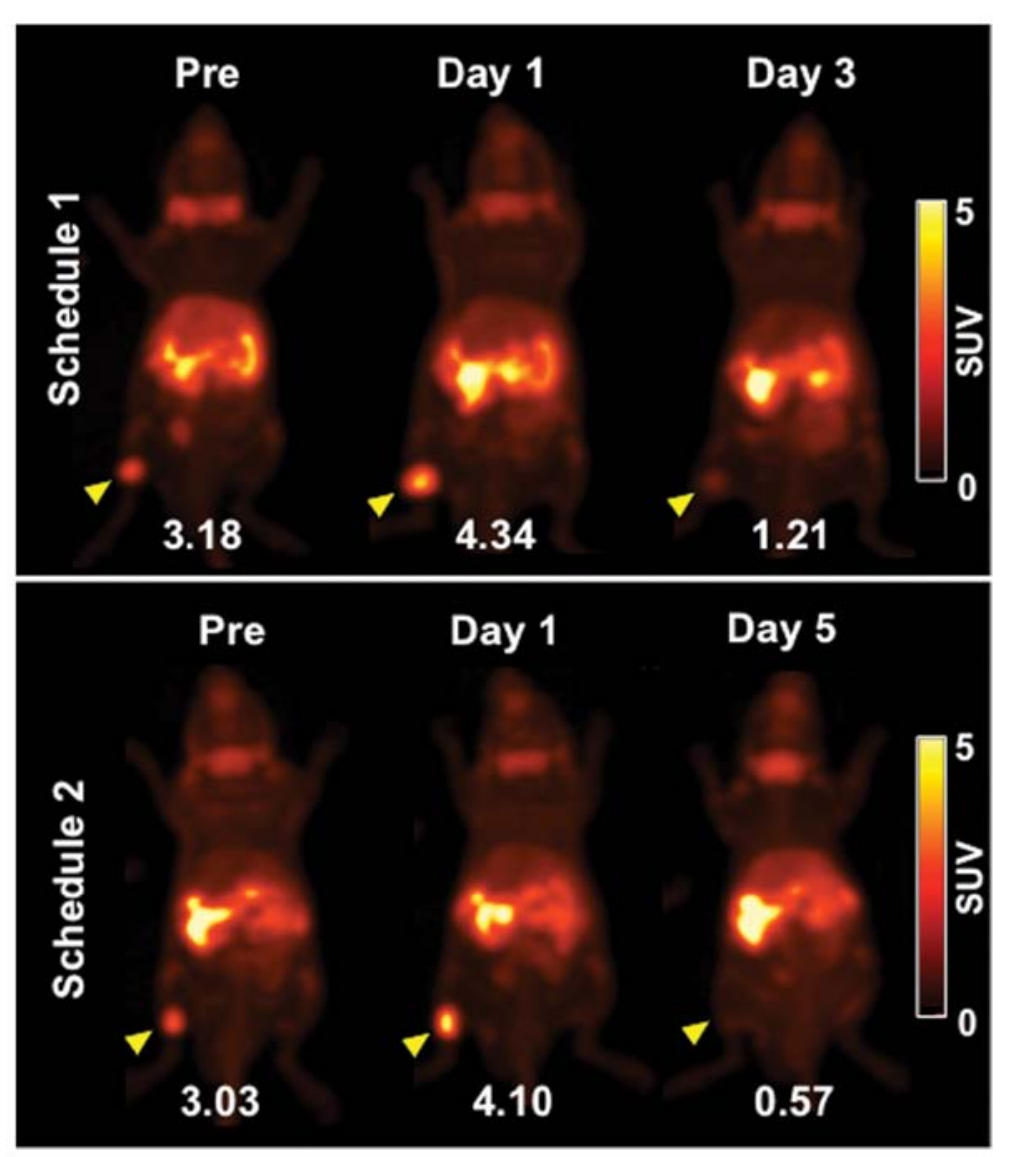

Figure 2. Representative serial PET images (maximum intensity projection) in schedules 1 (upper panel) and 2 (lower panel). The 10-min emission scans were conducted at $30 \mathrm{~min}$ after intravenous injection of $\sim 11.1 \mathrm{MBq}$ of $\left[3{ }^{-1} \mathrm{C}\right] \mathrm{AIB}$. Arrowheads indicate tumors. SUVmax values are shown under the images. SUV, standardized uptake value; PET, positron emission tomography.

Statistical analysis. Tumor uptake, positive cells of TUNEL or Ki-67, and mRNA expression data were analyzed by one-way ANOVA or two-way repeated-measures ANOVA, followed by the Student-Newman-Keuls multiple comparison test. The correlation between tumor uptake and other factors was examined by simple regression analysis. A value of $\mathrm{P}<0.05$ was considered statistically significant.

\section{Results}

Serial PET before and after X-ray irradiation. To determine the schedule of PET studies after X-irradiation, we measured the tumor sizes after $15 \mathrm{~Gy}$ radiation. Tumor sizes increased up to 2 days after irradiation and then decreased thereafter (Fig. 1 upper panel). All tumors had almost disappeared around 10 days after irradiation (Fig. 1 upper panel). $\left[{ }^{18} \mathrm{~F}\right] \mathrm{FDG}$ tumor uptake is reported to increase temporarily at early time-points (4 $\mathrm{h}$ to several days) after radiotherapy (12-14). As it is important to determine the change in $\left[3-{ }^{11} \mathrm{C}\right] \mathrm{AIB}$ tumor uptake at early time-points after irradiation, we selected day 1 for the first post-radiation PET study. We additionally selected two time-points, days 3 and 5, to evaluate change in tumor uptake on the way to tumor shrinkage. Due to the availability of the PET machine (5 days/week), we set two schedules of PET study, schedules 1 and 2, as shown in Fig. 1 (lower panel).

Representative PET images (maximum intensity projection) of $\left[3{ }^{-11} \mathrm{C}\right]$ AIB before (day -1$)$ and after irradiation are shown in Fig. 2. [3- $\left.{ }^{11} \mathrm{C}\right] \mathrm{AIB}$ PET clearly visualized tumors on day -1 and more clearly on day 1 (Fig. 2). Tumor uptake of $\left[3-{ }^{11} \mathrm{C}\right] \mathrm{AIB}$ on day 3 was markedly decreased and that on day 5 was barely detected (Fig. 2). Serial changes in tumor volume of the mice used for the PET studies are shown in Fig. 3 (upper panels). The volume of all tumors was increased on day 1 compared with that on day 0 , although the change was slight in three tumors (Fig. 3, upper panels). The volume of two tumors on day 3 in schedule 1 increased, while that of the remaining tumors decreased (Fig. 3, upper panels). On day 5, a great reduction in volume was observed in all tumors (Fig. 3, upper panels). Temporal changes in tumor uptake are shown in Fig. 3 (middle panels). Although tumor uptake was increased on day 1 , with the exception of two mice, there was no significant difference in mean SUVmax compared with that on day -1 (Fig. 3, middle panels). On days 3 and 5, tumor uptake was significantly decreased compared with that on day $-1(\mathrm{P}<0.05$ for day 3 and $\mathrm{P}<0.01$ for day 5$)$ (Fig. 3, middle panels). There was no correlation between tumor volume and uptake in schedule 1 (Fig. 3, lower left panel). Although there was a correlation in schedule 2 (Fig. 3, lower right panel), the correlation was lost when the data of day 5 were excluded (data not shown).

Histological analysis. Many mitotic cells were observed in the H\&E-stained sections of the untreated tumors (Fig. 4A, left column). The number of mitotic cells was markedly 

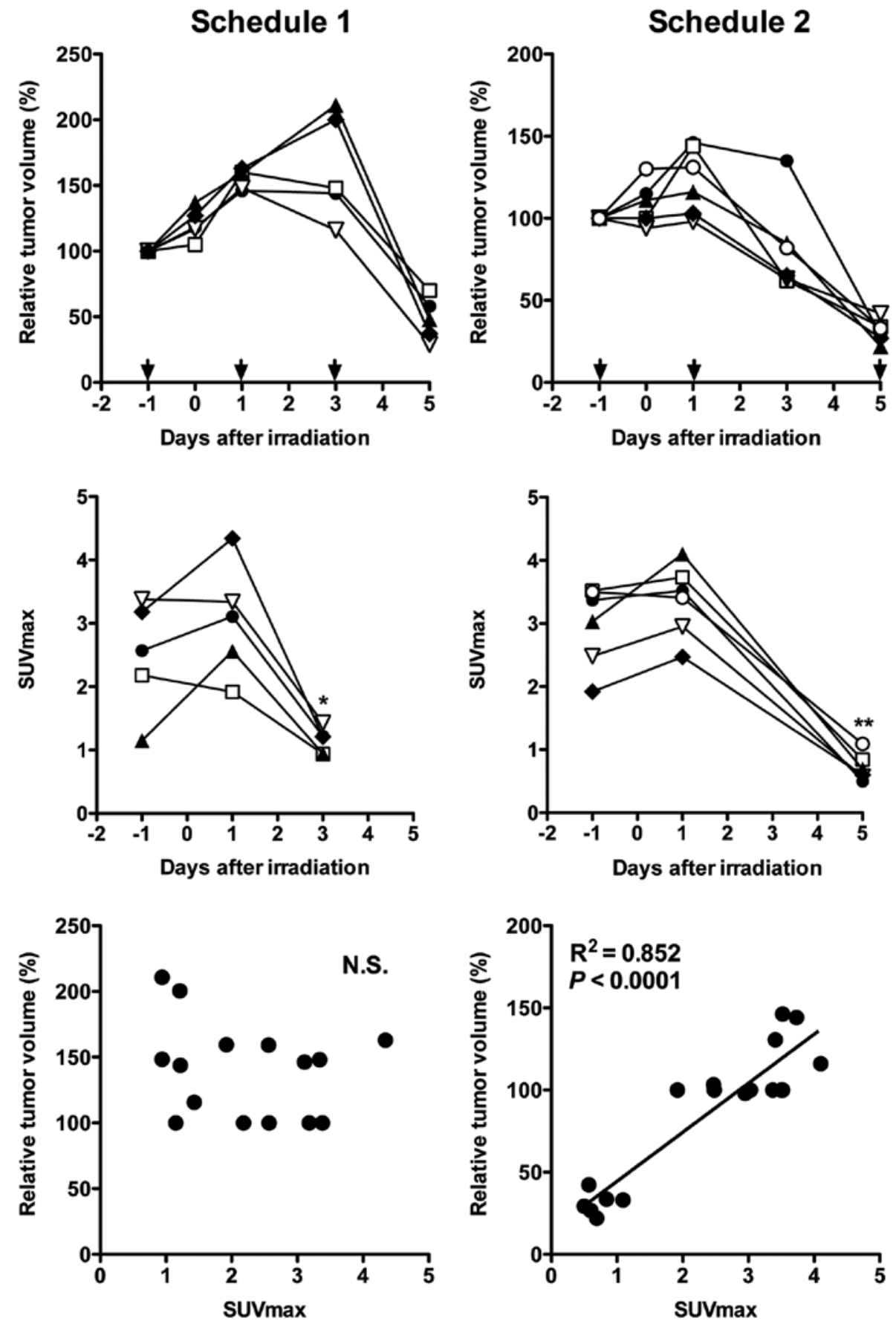

Figure 3. Tumor growth curve (upper panels), tumor uptake (middle panels), and correlation between tumor uptake and volume (lower panels) in schedules 1 (left column) and 2 (right column). Arrows indicate PET experiments in the upper panels. Tumor uptake is expressed as SUVmax. Data represent each replicate. ${ }^{* *} \mathrm{P}<0.01,{ }^{*} \mathrm{P}<0.05$ vs. pre-irradiation (day -1). N.S., no significant difference. PET, positron emission tomography.

decreased on day 1 (Fig. 4A, left column). On day 3, although the mitotic cells increased, necrotic cells and fibrosis were observed (Fig. 4A, left column). On day 5, few living cells were observed and fibrotic areas were widely spread (Fig. 4A, left column). Tumor sections were also stained for TUNEL and Ki-67 (Fig. 4A, middle and right columns), and the percentages of positive cells were quantified. We were not able to quantify the results of day 5 since only a few living cells were observed at this time-point, as described above. In the untreated sections stained with TUNEL, few TUNEL-positive (apoptotic) cells $(<0.5 \%)$ were observed (Fig. 4A, middle column and Fig. 4B, left panel). X-irradiation-induced apoptotic cells were $\sim 1.4 \%$ on day 1 and increased to $8 \%$ on day 3 (Fig. 4B, left panel). Although there was no significant difference between $0 \mathrm{~Gy}$ and day 1 , there was a significant difference between $0 \mathrm{~Gy}$ and day $3(\mathrm{P}<0.01)$. In the $\mathrm{Ki}-67$-stained sections, $\sim 20 \%$ of untreated cells were positively stained (Fig. 4A, right column and $4 \mathrm{~B}$, right panel). Although $\mathrm{Ki}-67$-positive cells were significantly decreased to $\sim 7 \%$ on day 1 ( $\mathrm{P}<0.01$ vs. $0 \mathrm{~Gy}$ ), they were increased to $\sim 20 \%$ on day 3 , which was as high as that in the untreated tumors (Fig. 4B, right panel). There were no significant correlations between $\left[3{ }^{-1} \mathrm{C}\right] \mathrm{AIB}$ tumor uptake, and percentages of TUNEL- or Ki-67-positive cells (data not shown). 
A

A H\&E TUNEL
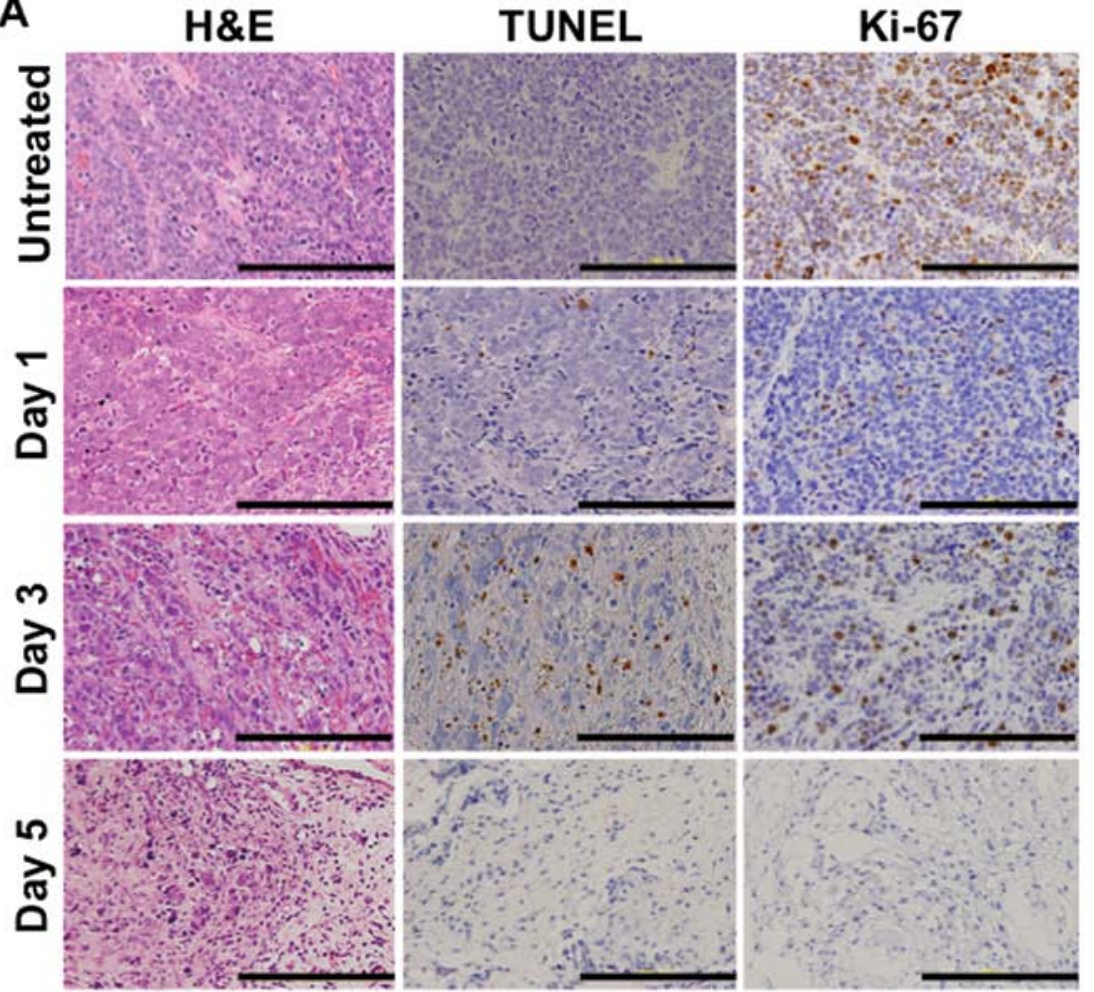

B
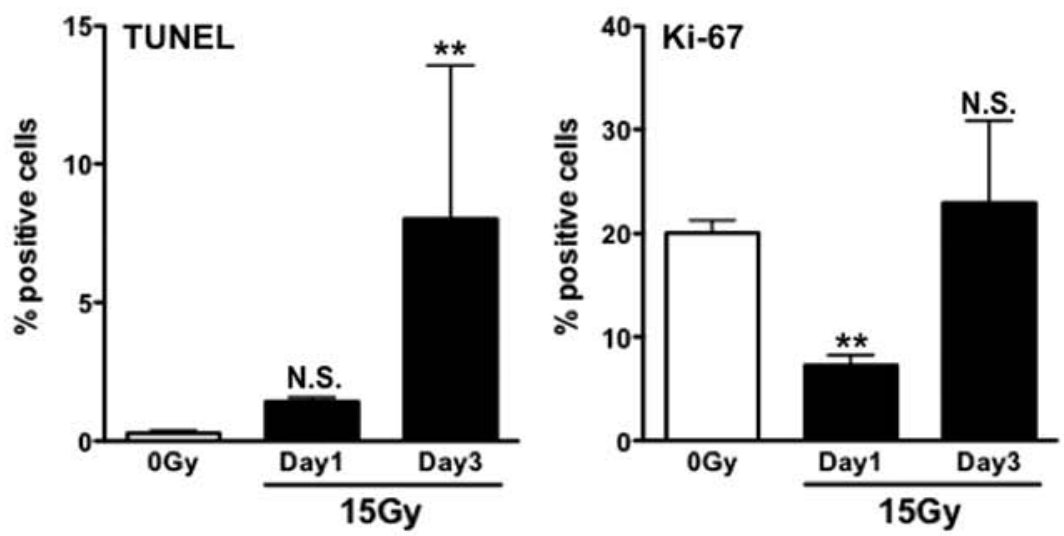

Figure 4. Histological analyses of tumors untreated and treated with radiation. (A) Representative images of H\&E- (left column), TUNEL-, and Ki-67-stained (right column) tumors. Scale bars, $200 \mu \mathrm{m}$. (B) Quantitative analysis of TUNEL- (left panel) and Ki-67-positive (right panel) cells. Data are expressed as the mean \pm SD. * $\mathrm{P}<0.05$ vs. 0 Gy. TUNEL, terminal deoxynucleotidyl transferase-mediated deoxyuridine triphosphate nick-end labeling; H\&E, hematoxylin and eosin.

Amino acid transporter expression analysis. The amino acid transporter system A consists of three transporters SLC38A1, SLC38A2, and SLC38A4 $(8,15,16)$. According to mRNA expression analysis of these three transporters by real-time RT-PCR, expression levels of both SLC38A1 and SLC38A2 were detected in the tumors (Fig. 5, upper panels), but not that of SLC38A4 (data not shown). The expression of SLC38A1 on day 1 tended to be increased compared with that with $0 \mathrm{~Gy}$, although there was no significant difference, and it decreased on days 3 and 5 with significant differences ( $\mathrm{P}<0.05$ vs. $0 \mathrm{~Gy}$; Fig. 5, upper left panel). That of SLC38A2 on day 1 was nearly equal to that with 0 Gy and decreased on days 3 and 5 with significant differences $(\mathrm{P}<0.05$ vs. 0 Gy; Fig. 5, upper right panel). There was a significant correlation between $\left[3-{ }^{11} \mathrm{C}\right] \mathrm{AIB}$ tumor uptake and SLC38A1 expression, but not SLC38A2 (Fig. 5, middle and lower panels).

\section{Discussion}

In the present study, to determine whether $\left[3{ }^{11} \mathrm{C}\right] \mathrm{AIB}$ is useful to monitor early metabolic change of tumors after radiotherapy, we conducted PET studies evaluating the tumor uptake change after irradiation in a tumor mouse model on two different schedules. One day after irradiation (day 1), the tumor uptake of $\left[3-^{11} \mathrm{C}\right] \mathrm{AIB}$ tended to increase, although there were no statistically significant differences compared with that one day before irradiation (day -1). This increased uptake was transient and decreased thereafter. Temporarily increased tumor uptake at early time-points ( $4 \mathrm{~h}$ to several days) after irradiation has also been observed in $\left[{ }^{18} \mathrm{~F}\right] \mathrm{FDG}$ PET of patients (12-14). Although this mechanism has not been clarified yet, there are two possible reasons; the increased uptake may be caused by an elevated inflammatory reaction or by 

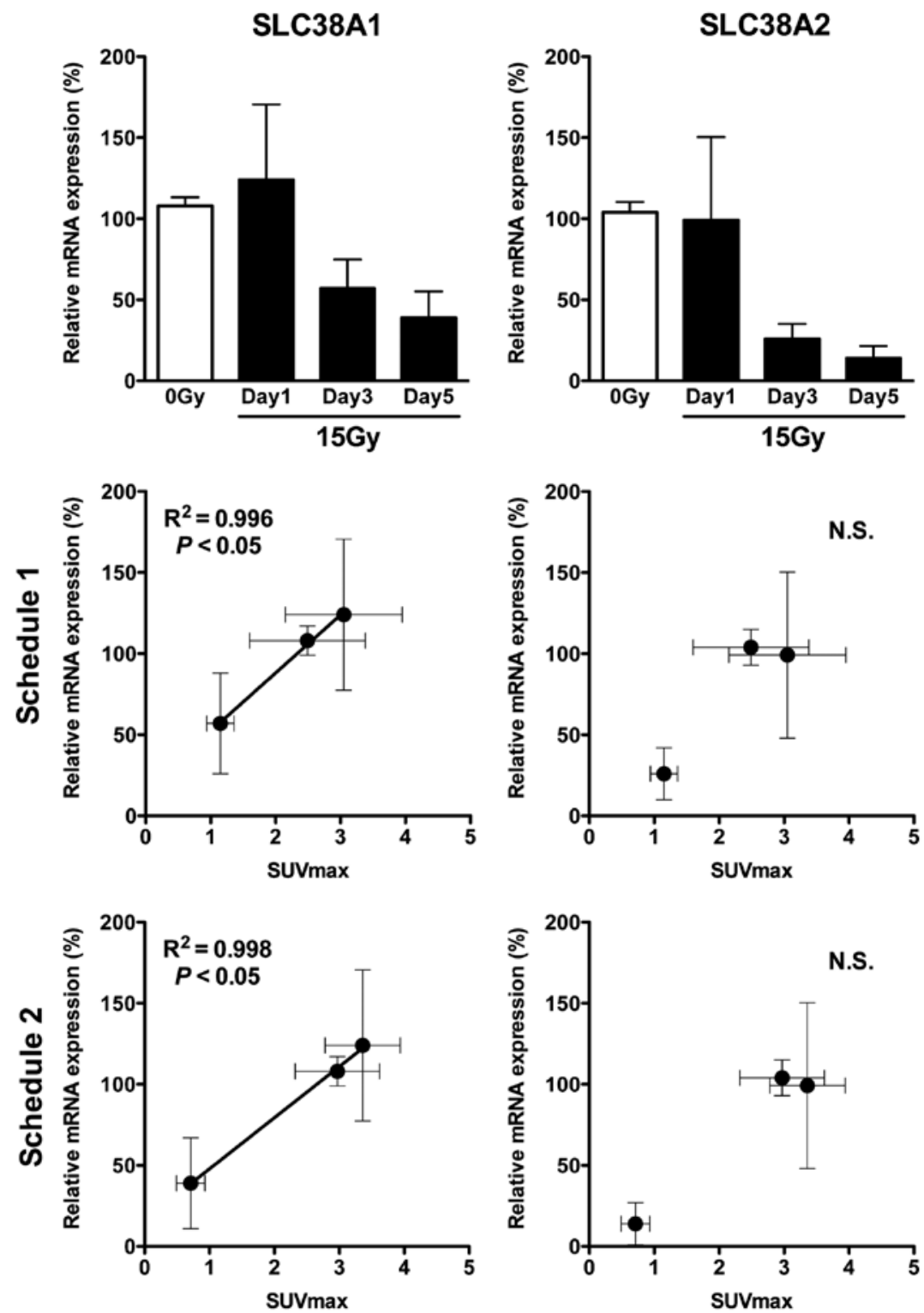

Figure 5. Amino acid transporter mRNA expression (upper panels) and correlation between tumor uptake and amino acid transporter expression in schedules 1 (middle panels) and 2 (lower panels). Those for SLC38A1 and SLC38A2 are shown in the left and right columns, respectively. Data are expressed as the mean \pm SD. "P<0.05 vs. 0 Gy. N.S. means no significant difference.

transiently enhanced glucose metabolism (12-14). [3- $\left.{ }^{11} \mathrm{C}\right] \mathrm{AIB}$ was reported to accumulate less in inflammatory lesions compared with $\left[{ }^{18} \mathrm{~F}\right] \mathrm{FDG}$ in a mouse model with acute inflammation (9). Furthermore, few inflammatory infiltrates were observed in the tumor sections in the present study. Hence, we can exclude the possibility that the increased tumor uptake of $\left[3-{ }^{11} \mathrm{C}\right] \mathrm{AIB}$ on day 1 was caused by induced inflammatory reaction, and the increased uptake is thought to reflect other pathophysiological change(s). Although the clinical implication of temporarily increased uptake of $\left[{ }^{18} \mathrm{~F}\right] \mathrm{FDG}$ early after radiotherapy is still unclear, it could be a predictor of favorable outcome of radiotherapy in brain tumors (13). In xenograft tumor models, increased $\left[{ }^{18} \mathrm{~F}\right] \mathrm{FDG}$ uptake and apoptotic cells were observed in a tumor with high radiosensitivity, but not in tumors with lower radiosensitivity (17). Since the present study was conducted using a single effective radiation dose for a single tumor model, further studies using tumor models with various radiosensitivities are needed to determine whether temporarily increased $\left[3-{ }^{11} \mathrm{C}\right] \mathrm{AIB}$ uptake after radiotherapy is related to the efficacy of radiotherapy.

For schedule 1, there was no correlation between tumor uptake and volume. For schedule 2, although there was a positive correlation between tumor uptake and volume, the tumor volume on day 5 was greatly decreased. This greatly decreased tumor uptake could be caused by the drastic reduction in viable cells as shown in the H\&E-stained sections on day 5 . When the data of day 5 were eliminated, there was no correlation between tumor uptake and volume for schedule 2 . These findings raise the possibility that a temporal change in tumor uptake by irradiation is independent of tumor volume at the earlier time-points. Although decreased tumor uptake of $\left[{ }^{18} \mathrm{~F}\right] \mathrm{FDG}$ after effective therapy provides useful information for the evaluation of efficacy, in general it is necessary to wait several months after radiotherapy to minimize the influence of radiation-induced inflammation. Our previous studies showed that the accumulation of $\left[3-{ }^{11} \mathrm{C}\right] \mathrm{AIB}$ was less in an inflammatory 
lesion compared with $\left[{ }^{18} \mathrm{~F}\right] \mathrm{FDG}$ in a mouse model with acute inflammation (9) as mentioned above. Therefore, $\left[3-{ }^{11} \mathrm{C}\right] \mathrm{AIB}$ PET has the potential for evaluating the response to radiotherapy at earlier time-points than $\left[{ }^{18} \mathrm{~F}\right] \mathrm{FDG}$ PET. Although $\left[3-{ }^{11} \mathrm{C}\right] \mathrm{AIB}$ tumor uptake was higher than $\left[{ }^{18} \mathrm{~F}\right] \mathrm{FDG}$ in tumorbearing mice $(9,10),\left[{ }^{18} \mathrm{~F}\right] \mathrm{FDG}$ tumor uptake is generally higher than the amino acid-based PET probes, such as [methyl- ${ }^{11} \mathrm{C}$ ] methionine, in patients. Considering the high sensitivity of $\left[{ }^{18} \mathrm{~F}\right] \mathrm{FDG}$ in detecting tumors in patients, combined PET of both $\left[{ }^{18} \mathrm{~F}\right] \mathrm{FDG}$ and $\left[3-{ }^{11} \mathrm{C}\right] \mathrm{AIB}$ may be clinically superior to [3- $\left.{ }^{11} \mathrm{C}\right] \mathrm{AIB}$ PET alone for increased sensitivity and to precisely evaluate the therapeutic efficacy. Further clinical studies are needed to assess the role of $\left[3-{ }^{11} \mathrm{C}\right] \mathrm{AIB}$ PET in the evaluation of therapeutic efficacy.

To explore the factor(s) responsible for $\left[3-{ }^{11} \mathrm{C}\right] \mathrm{AIB}$ tumor uptake change after irradiation, we conducted histological and expression analyses of tumors before and after irradiation. Based on the findings of the H\&E-stained sections, tumor cells transiently stopped proliferation early after irradiation and then started to proliferate again, and then cell death was induced. These findings were consistent with the analysis of the TUNEL- and Ki-67-stained sections in the present study. Although radiation-induced cell death occurred following cell regrowth after temporary cell cycle arrest in the irradiated SY cells in the present study as well as in other cells (1), the tumor uptake was not correlated with either apoptotic or proliferative cells. These findings suggest that $\left[3-{ }^{11} \mathrm{C}\right] \mathrm{AIB}$ PET could lead to fewer false-positive results due to transient cell regrowth activity as well as inflammation reaction. AIB is incorporated into cells mainly through amino acid transporter system A that consists of three transporters SLC38A1, SLC38A2 and SLC38A4 $(8,15,16)$. In the expression analysis, SLC38A1 and SLC38A2 were detected in the tumors, but SLC38A4 was not detected. Some proteins, including amino acid transporters, often show ectopic expression in malignant transformed cells $(8,15,16)$. Although SLC38A2 is expressed in normal lung, SLC38A1 and SLC38A4 are not expressed $(15,16)$. The SLC38A1 expression in SY tumors could be caused by oncogenic transformation. The SLC38A1 expression showed a tendency to increase temporarily on day 1, but not SLC38A2, and the expression of both decreased thereafter. The temporal change in tumor uptake of $\left[3-{ }^{11} \mathrm{C}\right] \mathrm{AIB}$ was correlated with that of SLC38A1 expression, but not with SLC38A2. The ectopic expression of SLC38A1 may play a major role in the high uptake of $\left[3-{ }^{11} \mathrm{C}\right] \mathrm{AIB}$ in SY tumors. This may be the reason why the temporal change of $\left[3-{ }^{11} \mathrm{C}\right] \mathrm{AIB}$ uptake by irradiation was correlated with that of SLC38A1 expression. Since each type of cancer has a distinct expression pattern of amino acid transporters $(15,16)$, it would be important to investigate the relationship between $\left[3-{ }^{11} \mathrm{C}\right] \mathrm{AIB}$ uptake and amino acid transporter expression after irradiation in several tumor models to elucidate the implication of temporal change of $\left[3-{ }^{11} \mathrm{C}\right] \mathrm{AIB}$ after radiotherapy.

The present study showed that the temporal change in [3- $\left.{ }^{11} \mathrm{C}\right] \mathrm{AIB}$ tumor uptake at early time-points after irradiation was correlated with the amino acid transporter expression, but independent of tumor volume and cell proliferation activity, suggesting that $\left[3-{ }^{11} \mathrm{C}\right] \mathrm{AIB}$ PET has the potential for evaluating an early metabolic change after radiotherapy not affected by transient proliferation activity. Our findings support further preclinical and clinical studies to investigate the role of metabolic change detected by $\left[3-{ }^{11} \mathrm{C}\right] \mathrm{AIB}$ PET in the evaluation of radiotherapy efficacy before morphological change of tumors.

\section{Acknowledgements}

We thank Takamitsu Morioka for the expertise in assessing the histological findings; Yuriko Ogawa for technical assistance; Yuichiro Yoshida, Masanao Ogawa, and Nobuki Nengaki in the Radiochemistry section for technical support of $\left[3-{ }^{11} \mathrm{C}\right] \mathrm{AIB}$ production; staff in the Cyclotron Operation section for cyclotron operation; and staff in the Laboratory Animal Sciences section for animal management. The present study was supported by grants from the Ministry of Education, Culture, Sports, Science and Technology of Japan (KAKENHI 23510289 and 24591803).

\section{References}

1. Halperin EC, Perez CA and Brady LW: Perez and Brady's Principles and Practice of Radiation Oncology. 5th edition. Lippincott Williams \& Wilkins, Philadelphia, PA, 2008.

2. Van de Wiele C, Lahorte C, Oyen W, Boerman O, Goethals I, Slegers G and Dierckx RA: Nuclear medicine imaging to predict response to radiotherapy: a review. Int J Radiat Oncol Biol Phys 55: 5-15, 2003.

3. Juweid ME and Cheson BD: Positron-emission tomography and assessment of cancer therapy. N Engl J Med 354: 496-507, 2006.

4. Bading JR and Shields AF: Imaging of cell proliferation: status and prospects. J Nucl Med 49: 64S-80S, 2008.

5. Shreve PD, Anzai Y and Wahl RL: Pitfalls in oncologic diagnosis with FDG PET imaging: physiologic and benign variants. Radiographics 19: 61-77, 1999.

6. Love C, Tomas MB, Tronco GG and Palestro CJ: FDG PET of infection and inflammation. Radiographics 25: 1357-1368, 2005.

7. Haberkorn U, Strauss LG, Dimitrakopoulou A, et al: PET studies of fluorodeoxyglucose metabolism in patients with recurrent colorectal tumors receiving radiotherapy. J Nucl Med 32: 1485-1490, 1991.

8. McConathy $\mathbf{J}$ and Goodman MM: Non-natural amino acids for tumor imaging using positron emission tomography and single photon emission computed tomography. Cancer Metastasis Rev 27: 555-573, 2008.

9. Tsuji AB, Kato K, Sugyo A, et al: Comparison of 2-amino[3-11 C]isobutyric acid and 2-deoxy-2- $\left[{ }^{18} \mathrm{~F}\right]$ fluoro-D-glucose in nude mice with xenografted tumors and acute inflammation. Nucl Med Commun 33: 1058-1064, 2012.

10. KatoK,Tsuji AB,Saga T and Zhang M-R: An efficient and expedient method for the synthesis of ${ }^{11} \mathrm{C}$-labeled $\alpha$-aminoisobutyric acid: a tumor imaging agent potentially useful for cancer diagnosis. Bioorg Med Chem Lett 21: 2437-2440, 2011.

11. Yoshida C, Tsuji AB, Sudo H, et al: Therapeutic efficacy of c-kit-targeted radioimmunotherapy using 90Y-labeled anti-c-kit antibodies in a mouse model of small cell lung cancer. PLoS One 8: e59248, 2013.

12. Hautzel H and Müller-Gärtner HW: Early changes in fluorine-18-FDG uptake during radiotherapy. J Nucl Med 38: 1384-1386, 1997.

13. Maruyama I, Sadato N, Waki A, et al: Hyperacute changes in glucose metabolism of brain tumors after stereotactic radiosurgery: a PET study. J Nucl Med 40: 1085-1090, 1999.

14. Rozental JM, Levine RL, Mehta MP, et al: Early changes in tumor metabolism after treatment: the effects of stereotactic radiotherapy. Int J Radiat Oncol Biol Phys 20: 1053-1060, 1991.

15. Mackenzie B and Erickson JD: Sodium-coupled neutral amino acid (System N/A) transporters of the SLC38 gene family. Pflugers Arch 447: 784-795, 2004.

16. Nakanishi T and Tamai I: Solute carrier transporters as targets for drug delivery and pharmacological intervention for chemotherapy. J Pharm Sci 100: 3731-3750, 2011.

17. Furuta M, Hasegawa M, Hayakawa K, et al: Rapid rise in FDG uptake in an irradiated human tumour xenograft. Eur J Nucl Med 24: 435-438, 1997. 